\title{
Principles of Neurophysiological Assessment, Mapping, and Monitoring - Second Edition
}

\author{
Scott Francis Davis, Alan David Kaye (Editors). Springer; 2020; price: \$109.99 \\ (softcover); 316 pages. ISBN: 978-3-030-22399-1
}

\author{
Michale Ok $\cdot$ John McAuliffe
}

Received: 26 February 2020/Revised: 26 February 2020/Accepted: 26 February 2020/Published online: 23 March 2020

(C) Canadian Anesthesiologists' Society 2020

Intraoperative neurophysiological monitoring is now considered an essential component of the perioperative care of patients undergoing procedures that place neurological function at risk. To obtain the best outcomes, it is important that anesthesiologists, surgeons, and other proceduralists understand the principles governing neurophysiological monitoring modalities. A shared mental model would enable clinicians to make more informed decisions, ultimately leading to timely interventions. In the Principles of Neurophysiological Assessment, Mapping, and Monitoring, authors Davis and Kaye provide the necessary background for clinicians to understand the basics of neurophysiology and monitoring as well as the details of single modalities and their integration into specific procedures. This textbook can serve as a resource for both those seeking an introduction to the field and experienced practitioners.

The textbook is of modest length, with 316 pages, and is composed of 23 chapters, which can be grouped into three main sections: foundational concepts (Chapters 1-5), neurophysiological monitoring modalities (Chapters 611), and case-based application (Chapters 12-23). This carefully considered organization of the text is one of the book's primary strengths. Rather than making the reader skip to subsequent chapters for explanations of concepts not described earlier in the book, the chapters are organized to be read from the beginning, in order.

Because the target audience of this book is broad and likely includes personnel who work infrequently in the operating room, the first chapter begins by educating the

\footnotetext{
M. Ok · J. McAuliffe ( $₫)$

Cincinnati Children's Hospital Medical Center, Cincinnati, OH, USA

e-mail: john.mcauliffe@cchmc.org
}

reader about the operating room's environment and culture. Although there is a brief discussion of communication that mentions the role of the clinician conducting the neuromonitoring, there are no details on the context or methodology for critical communications in the operative environment. Thus, a full discussion regarding the communication of neurophysiological data to all members of the operative team would add to the value of the book. The second chapter discusses the relevant anatomy in detail, which is of great importance for accurately identifying the site of neurological injury during monitoring. Thoughtful, frequent use of figures and tables well complements this chapter. The next two chapters discuss cellular neurophysiology and electrophysiology concepts that are relevant to neurophysiological monitoring. The electrophysiology chapter is especially useful because it discusses how neural structures are stimulated and how the signals are acquired. For readers without an engineering background, the authors summarize the core principles that a neuromonitorist must understand and offer practical tips. For example, in the discussion on the concept of signal averaging, the authors provide a guide for computing optimal stimulation rates for minimizing 60-cycle noise and its harmonics. Additional guidance on the use of filtering is also provided.

The last topic covered in the foundational concepts section is the impact that modern anesthetics may have on various neurophysiological modalities. Many of the authors contributing to the book are practicing anesthesiologists and so here provide an in-depth analysis of various anesthetic agents and their corresponding effects on the monitoring process. For readers who are not anesthesiologists, this chapter will be particularly useful 
for understanding the effects of anesthetic agents on monitoring.

The second section of the book (Chapters 6-11) discuss individual neuromonitoring modalities in detail. The modalities include somatosensory-evoked potentials (SSEPs), motor-evoked potentials (MEPs), electromyography, brain-stem auditory evoked potentials, electroencephalography, and the H-reflex and F-response. Each chapter is composed of an introduction, the relevant development and history of the modality, stimulation means, recording the signals, and parameters. Toward the end of each chapter, the authors propose useful interpretation guidelines and corresponding alarm criteria. Because some modalities do not have universally accepted alarm criteria, the variously accepted criteria are presented (e.g., in the transcranial MEP chapter). Although not commonly utilized in the operating room, visual-evoked potentials seemingly did not merit a dedicated chapter. Including a discussion on visual-evoked potentials and some other topics, such as the bulbocavernous reflex, would round out the chapter's content, making it a more complete reference for neurophysiological monitoring modalities.

Chapter 11 explains why, in addition to SSEPs and MEPs, the H-reflex and F-response can be useful. The authors carefully explain that SSEP and MEP recordings monitor central function but do not address other important aspects of spinal cord integrity. A detailed explanation of how an H-reflex, seen on electromyography, can be useful when evaluating spinal cord reflexes analogous to stretch reflexes utilizing electric stimulation. They offer practical instruction, with the use of illustrations, on how to provoke and capture the H-reflex, including electrode placement, the commonly used muscle groups, and the recording sites.

The final section of the book (Chapters 12-23) focuses on practical application of neurophysiological monitoring.
This section can be divided into two subsections. The first subsection, which includes Chapters 12-18, addresses strategies for monitoring specific operative categories, including spine surgery, spinal cord surgery, posterior fossa craniotomy, carotid endarterectomy, and head and neck procedures. Chapters 13 and 18 discuss mapping techniques for the spinal cord and cortex. The second subsection is what could be categorized as "special monitoring applications." These special situations include pediatric neuromonitoring, assessing spinal cord nerve roots, and application of pain medicine.

In Principles of Neurophysiological Assessment, Mapping, and Monitoring, authors Davis and Kaye have written a textbook that is succinct, comprehensive, and, most importantly, practical for daily application. We believe that it is an excellent textbook for novice neurophysiological monitorists because it is easy to read thanks to its logical organization. In addition, previous neuromonitoring textbooks have tended to be more theoretical than practical. The authors of Principles, in contrast, have included practical suggestions for monitoring parameters and troubleshooting common problems. In addition, readers who are pursuing certification should be grateful to the authors for pointing out commonly tested themes. Overall, it is an excellent reference for an increasingly wide audience, from the novice to the expert.

\section{Conflicts of interest None.}

Funding statement None.

Editorial responsibility This submission was handled by Dr. Hilary P. Grocott, Editor-in-Chief, Canadian Journal of Anesthesia.

Publisher's Note Springer Nature remains neutral with regard to jurisdictional claims in published maps and institutional affiliations. 